Francisco Antonio Cabaleiro - María Jesús Sainz · Socorro

Seoane-Labandeira · María Elvira López-Mosquera

\title{
Estiércol de pollo peletizado: potencial fertilizante inmediato y residual en cultivo de lechuga
}

Recibido: 3 outubro 2017 / Aceptado: 21 decembro 2017

\begin{abstract}
Resumen Se evaluó el potencial fertilizante de un estiércol deshidratado y granulado de pollo (Broiler Litter, BL) en un cultivo de lechuga en invernadero. Se establecieron dos ensayos, uno en el periodo otoño-invierno, y otro en primavera. Se aplicaron distintos tratamientos fertilizantes: parcelas no fertilizadas (Control), fertilizadas con nitrato amónico (M, $120 \mathrm{~kg} \mathrm{~N}^{-1}$ ), fertilizadas con un fertilizante mineral de liberación lenta $\left(\mathrm{Mr}, 120 \mathrm{~kg} \mathrm{~N} \mathrm{ha}^{-1}\right)$ y con dosis crecientes de $B L$ para satisfacer las necesidades de $\mathrm{N}$ de la lechuga $\left(60,80,120\right.$ y $240 \mathrm{~kg} \mathrm{~N}^{-1}$ en otoño-invierno y 120,240 y $360 \mathrm{~kg} \mathrm{~N}^{-1}$ en primavera). El abono se incorporó al comienzo de ambos ensayos, en una sola aplicación, lo que permitió estudiar el efecto residual en una segunda cosecha para ambas épocas. En los dos ensayos, de otoño-invierno y de primavera, la fertilización con BL, a cualquiera de las dosis aplicadas, garantizó la producción del cultivo de lechuga, del mismo modo o incluso superior al abonado mineral. El fertilizante $\mathrm{BL}$ se comportó como un abono de liberación gradual, proporcionando una cantidad importante de nitrógeno para las segundas cosechas, fruto del efecto residual del abonado aplicado dos meses antes. La fertilización con BL en dosis superiores a $120 \mathrm{~kg}$ de $\mathrm{N}$ ha${ }^{1}$ para el periodo otoño-invierno y primavera suministraron
\end{abstract}

Francisco Cabaleiro · Elvira López-Mosquera Institute of Agricultural Biodiversity and Rural Development (IBADER), University of Santiago de Compostela, E-27002 Lugo, Spain

María Jesús Sainz

Department of Plant Production, University of Santiago de Compostela, E-27002 Lugo, Spain

Socorro Seoane-Labandeira

Department of Soil Science, University of Santiago de

Compostela, Spain

Tel.:+34 982223134

e-mail: franciscoantonio.cabaleiro@gmail.com nutrientes suficientes para obtener un segundo ciclo con un alto porcentaje de lechugas comerciales.

Palabras Clave estiércol de aves, fertilización orgánica, Lactuca sativa L., rendimiento del cultivo.

Pelletized broiler litter: Immediate and residual fertilizing potential in lettuce culture

Abstract The fertilizing potential of granulated dehydrated broiler litter (BL) for greenhouse cultivation of lettuce was assessed. Two different experiments were conducted in autumn-winter and spring. Fertilizer treatments included: no fertilization (Control) and application of either ammonium nitrate $\left(\mathrm{M}, 120 \mathrm{~kg} \mathrm{~N}^{-1}\right)$, a slow-release mineral fertilizer (Mr, $120 \mathrm{~kg} \mathrm{~N} \mathrm{ha}^{-1}$ ) and increasing rates of $\mathrm{BL}(60,80,120$ y $240 \mathrm{~kg} \mathrm{~N} \mathrm{ha}^{-1}$ in autumn-winter and 120, 240 y $360 \mathrm{~kg} \mathrm{~N}$ $\mathrm{ha}^{-1}$ in spring). The fertilizers were applied once at the start of each period, which allowed the residual effect on a second crop to be also assessed. Fertilization with BL at any rate ensured production of lettuce with yields similar to or even higher than fertilization with the other products. The BL fertilizer behaved as a gradual release fertilizer, providing a significant amount of nitrogen for the second crops as a result of the residual effect of the fertilizer applied two months before. The fertilization with $\mathrm{BL}$ in doses higher than $120 \mathrm{~kg}$ of $\mathrm{N} \mathrm{ha}^{-1}$ for the autumn-winter and spring period provided sufficient nutrients to obtain a second cycle with a high percentage of commercial lettuces.

Keywords Poultry manure, organic fertilization, Lactuca sativa L., crop yield.

\section{Introducción}

En las últimas décadas en la Europa de los 28 se ha producido una intensificación de la producción ganadera, que ha originado un incremento en la cantidad de estiércoles, cuya gestión exige estrategias de manejo que minimicen su impacto ambiental. En 2003, se produjeron en España 42.085 miles de toneladas de estiércol de bovino, 25.242 de porcino y 7.695 de aves (MAGRAMA, 2012). El 
principal destino de estos estiércoles sigue siendo la aplicación en campo con fines agrícolas, utilizándolos como enmiendas y abonos orgánicos.

El estiércol de pollo de engorde se considera un buen fertilizante, ya que es capaz de suministrar los macronutrientes ( $N, P$ y $K$ ) que necesitan las plantas (Tewolde, 2005a, b). Además, aumenta el contenido en carbono del suelo (Roberson et al. 2008) mejorando así sus propiedades físicas, químicas y biológicas (Gascho \& Hubbard, 2006; Ghanbarian et al. 2008; Pratt \& Tewolde, 2009) así como el rendimiento de los cultivos (Adeli et al. 2005; Tewolde et al. 2007).

Sin embargo, la gestión del estiércol en las granjas de pollos de engorde (Gallus gallus domesticus) presenta dificultades de almacenamiento ya que, debido a los cortos ciclos de producción animal, se genera estiércol a lo largo de todo el año, mientras que la fertilización de los cultivos se realiza fundamentalmente en primavera y otoño, por lo que este material puede permanecer acumulado durante periodos prolongados en la explotación. Durante su almacenamiento se producen pérdidas de $\mathrm{N}$ por volatilización en forma de $\mathrm{N}$ amoniacal hacia la atmósfera y de K por lixiviación (Beegle \& Bosworth, 1997).

El proceso de deshidratación y granulación del estiércol de pollo permite reducir los problemas que origina su almacenamiento en fresco convirtiéndolo en un abono libre de patógenos y restos de antibióticos, que no desprende mal olor y se almacena, transporta y aplica fácilmente (López-Mosquera et al. 2008). En sistemas de cultivo intensivo bajo invernadero o en cultivo ecológico se requieren abonos orgánicos de calidad, entendiendo por ello que aporten los nutrientes necesarios para el cultivo de forma gradual, sin riesgo de que introduzcan patógenos, semillas de adventicias, o incrementen el nivel de elementos potencialmente tóxicos (metales y/o sales).

En 2012, la superficie cultivada de lechuga en España fue de 33.196 ha, localizadas principalmente en regadío al aire libre en la Región de Murcia (14.065 ha) y en Andalucía (11.415 ha) (MAGRAMA, 2012), zonas donde la intensificación de la agricultura está determinando un deterioro de las propiedades físicas del suelo.

La lechuga, al igual que otros cultivos aprovechables por sus hojas, precisan de un gran aporte de nitrógeno, acentuándose las necesidades de éste en la parte final de su ciclo (Broadley et al. 2003) correspondiendo con el inicio del acogollado. En esta etapa, la lechuga absorbe alrededor del 60-65 \% de todos los nutrientes. Esto pone de manifiesto la importancia del empleo de fertilizantes de liberación lenta, como puede ser el BL.

El objetivo de este trabajo fue estudiar los efectos de diferentes dosis de un estiércol deshidratado y granulado de pollo (BL) sobre la producción de lechuga en invernadero en dos épocas de cultivo diferenciadas, tanto en primavera como en otoño-invierno, en comparación con el abonado mineral convencional. También se evaluó el efecto fertilizante residual de este abono en cada época de cultivo para una segunda cosecha sin fertilizar.

\section{Material y Métodos}

Los ensayos se establecieron en invernadero sobre un Umbrisol húmico, desarrollado sobre esquistos cuarcíticos, con un $\mathrm{pH}$ ligeramente ácido $(6,3)$, adecuado contenido en M.O. (6,0 \%), alta relación $\mathrm{C} / \mathrm{N}(16,5)$, elevados niveles de $\mathrm{P}$ y K disponibles $\left(106,4 \mathrm{mg} \mathrm{kg}^{-1}\right.$ y $292,5 \mathrm{mg} \mathrm{kg}^{-1}$ respectivamente) y con bajo nivel de sales y metales.

En bancales de $6 \times 1 \mathrm{~m}^{2}$ y $0,3 \mathrm{~m}$ de altura se aplicaron al azar seis tratamientos fertilizantes ( 3 bancales por tratamiento): control sin fertilizar (C), aplicación de $58,5 \mathrm{~g} \mathrm{~m}^{-}$ 2 de nitrato amónico $(20,5 \%$ de N) para suministrar $120 \mathrm{~kg}$ $\mathrm{N} \mathrm{ha}^{-1}(\mathrm{M})$, y aplicación de 4 dosis del estiércol deshidratado y granulado $\mathrm{BL}\left(266,7,366,7,532,0\right.$ y $1064,0 \mathrm{~g} \mathrm{~m}^{-2}$, para proporcionar respectivamente $60,80,120$ y $240 \mathrm{~kg} \mathrm{~N}^{-1}$ (BL1, BL2, BL3 y BL4)); las dosis se calcularon en función del $\mathrm{N}$, teniendo en cuenta un porcentaje de mineralización del estiércol de pollo del $60 \%$ (Kissel, 2008) y que la riqueza de la partida del $\mathrm{BL}$ utilizado contenía un $4,2 \%$ de $\mathrm{N}, \mathrm{C} / \mathrm{N} 7,7, \mathrm{P}_{2} \mathrm{O}_{5} 6,1 \%, \mathrm{~K}_{2} \mathrm{O} 4,8 \%$. Los abonos se distribuyeron de forma manual y se incorporando al suelo con una labor poco profunda.

El suelo se mantuvo con un potencial hídrico entre 0,02 y $0,025 \mathrm{MPa}$ a través de riego por goteo. Los bancales fueron acolchados con una película de polietileno negro de 0,025 $\mathrm{mm}$ de espesor para evitar el desarrollo de adventicias.

En el mes de octubre se llevó a cabo la plantación de lechuga del primer ciclo otoño-invierno, estableciendo en cada bancal 60 plántulas de 3-4 hojas, en triple hilera, del cultivar 'Santa Cruz' (casa comercial Asgrow), lechuga acogollada tipo 'Trocadero', que se recolectaron en noviembre, 49 días después de su plantación.

Un mes más tarde, en diciembre, se plantó el segundo ciclo de lechuga con plántulas del cultivar Plenty (casa comercial Asgrow), una variedad de invierno cuyas características son muy parecidas a la variedad 'Santa Cruz' pero con mejor tolerancia a las bajas temperaturas. En este caso no se hizo ningún abonado adicional. Las lechugas de este segundo ciclo de cultivo se recogieron en febrero, 62 días después de la plantación.

Las bajas temperaturas registradas en este segundo ciclo de lechuga obligaron a emplear una manta térmica, que se utilizó por la noche y se retiró por el día, hasta que las temperaturas fueron adecuadas para el cultivo.

En marzo, manteniendo los mismos bancales, instalación de riego por goteo y acolchado que en los cultivos de otoñoinvierno, se diseñó un segundo ensayo para estudiar el potencial fertilizante a corto plazo y residual de tres dosis del estiércol deshidratado y granulado BL (BL3, BL4 y BL5) en el cultivo de lechuga, en comparación con un control no abonado (C) y dos tratamientos minerales ( $\mathrm{M}$ y $\mathrm{Mr}$ ). Se llevaron a cabo para ello dos ciclos consecutivos.

Se mantuvo la misma distribución de tratamientos fertilizantes en los bancales que en el ensayo de otoñoinvierno, se siguió utilizando el fertilizante mineral nitrato

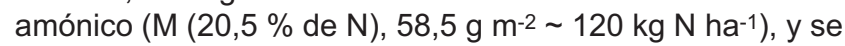
hizo uso de un nuevo fertilizante nitrogenado de liberación lenta $\left(\mathrm{Mr}\left(12 \% \mathrm{~N}, 12 \% \mathrm{P}_{2} \mathrm{O}_{5}, 17 \% \mathrm{~K}_{2} \mathrm{O}\right), 100 \mathrm{~g} \mathrm{~m}^{-2}\right)$, en los 
bancales donde inicialmente se había aplicado la dosis más baja de estiércol deshidratado y granulado BL (BL1), proporcionando $120 \mathrm{~kg} \mathrm{~N}$ ha-1. También se eliminó el tratamiento BL2, aunque se siguieron utilizando las dos dosis más altas de estiércol deshidratado y granulado $\mathrm{BL}$ (BL3, 634,7 $\mathrm{g} \mathrm{m}^{-2} \sim 120 \mathrm{~kg} \mathrm{~N}^{-1}$ y $^{-1}$ BL4, 1236,9 $\mathrm{g} \mathrm{m}^{-2} \sim 240$ $\mathrm{kg} \mathrm{N}$ ha-1), del ensayo de otoño-invierno. Se aplicó una nueva dosis de $\mathrm{BL}$ (BL5, 1904,0 $\mathrm{g} \mathrm{m}^{-2}$ ), incrementando el aporte de $\mathrm{N}$ hasta los $360 \mathrm{~kg} \mathrm{ha}^{-1}$, para intentar encontrar el techo de producción de este cultivo en primavera. La riqueza de la partida del $\mathrm{BL}$ utilizado en este segundo cultivo contenía un $3,7 \%$ de $\mathrm{N}, \mathrm{C} / \mathrm{N} 11,4,3,9 \%$ de $\mathrm{P}_{2} \mathrm{O}_{5}$ y $3,9 \%$ de $\mathrm{K}_{2} \mathrm{O}$.

Durante este segundo ensayo de primavera se instaló una malla de sombreo, con un calado del $50 \%$, colocada a modo de cubierta a $2,5 \mathrm{~m}$ de altura sobre el cultivo, para reducir la radiación directa y consecuentemente el calor y la temperatura sobre la planta de lechuga. El cultivar utilizado fue 'Santa Cruz', que se recolectó en abril, 45 días después de la plantación. En mayo se estableció un segundo ciclo de lechuga de primavera, sin hacer de abonado en ningún tratamiento, plantando el cultivar 'Hades' (casa comercial Asgrow), una variedad de verano cuyas características son muy parecidas a la variedad 'Santa Cruz' pero con resistencia al espigado, que se recolectó en junio, transcurridos 47 días desde que se estableció el cultivo.

En los cuatro ciclos de cultivo, las lechugas se recolectaron entre las 9 y las $12 \mathrm{~h}$ de la mañana. En cada ciclo, se pesaron en fresco las 60 lechugas de cada bancal. En ambos ensayos, y para cada ciclo de lechuga, se cuantificó en cada tratamiento el número de lechugas de valor comercial, es decir aquellas cuyo peso superaba los $100 \mathrm{~g}$, peso a partir del cual una lechuga tiene valor comercial (Reglamento (CE) № 1543/2001). Por diferencia con el número total de lechugas cosechadas, se obtuvo el número y el porcentaje de lechugas no comerciales de cada tratamiento, tanto para el primer ensayo (ciclos de otoñoinvierno), como para el segundo (ciclos de primavera).

Los datos se sometieron a un análisis de varianza con un solo factor, Anova I. Las medias se compararon mediante el test de la diferencia mínima significativa (DMS), comprobando si los datos eran normales (prueba de Kolmogorov-Smirnov) y efectuando la prueba de homogeneidad de la varianza de Levene. Cuando las varianzas no fueron homogéneas, se aplicó la prueba de Mann-Whithey. Se empleó el paquete software estadístico SPSS 20.0 .

Se estimó también la relación entre la dosis de $\mathrm{BL}$ y el peso fresco de la lechuga para el ciclo II de primavera, mediante un análisis de regresión, utilizando el menor valor de la varianza residual y el coeficiente de determinación $\left(R^{2}\right)$ como criterios para la selección de la ecuación con mejor bondad de ajuste.

\section{Resultados y Discusión}

\section{Producción de lechuga: cultivos de otoño-invierno}

En todos los tratamientos fertilizantes el peso medio de lechuga en fresco fue superior a los tratamientos control, que registraron 200 y $47 \mathrm{~g}$ por unidad de lechuga para el ciclo I y II respectivamente (figura 1). El peso medio más alto se obtuvo en el ciclo I con la dosis más elevada de $\mathrm{BL}$ (BL4: 1064,0 $\mathrm{g} \mathrm{m}^{-2} \sim 240 \mathrm{~kg} \mathrm{~N} \mathrm{ha}^{-1}$ ); en el ciclo II, las lechugas de mayor peso fueron de solo $150 \mathrm{~g} \mathrm{ud}^{-1}$ y se obtuvieron también en el tratamiento BL4 (figura 1).

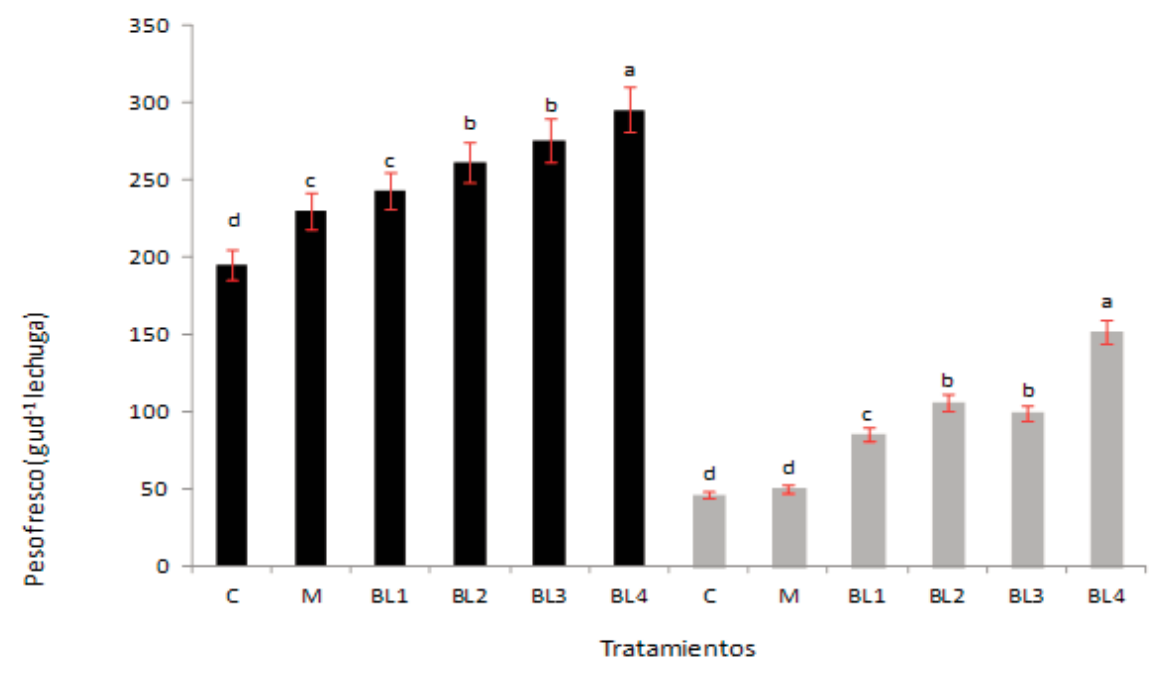

Ciclo I de otoño (noviembre)

Ciclo II de invierno (febrero)

Barras encabezadas por distintas letras indican diferencias significativas entre tratamientos para $p<0,05$.

Figura 1.- Peso fresco de lechuga (g ud-1 de lechuga) en los cultivos I y II de otoño-invierno. C: control; M: Nitrato amónico (58,5 $\left.\mathrm{g} \mathrm{m}^{-2} \sim 120 \mathrm{~kg} \mathrm{~N}^{-1}\right)$; estiércol deshidratado y granulado BL, BL1: $\left(266,7 \mathrm{~g} \mathrm{~m}^{-2} \sim 60 \mathrm{~kg} \mathrm{~N}^{-1}\right)$, BL2: $\left(366,7 \mathrm{~g} \mathrm{~m}^{-2} \sim 80 \mathrm{~kg} \mathrm{~N}^{-1}\right)$, BL3: $\left(532,0 \mathrm{~g} \mathrm{~m}^{-2} \sim 120 \mathrm{~kg} \mathrm{~N}^{-1}\right)$ y BL4: $\left(1064,0 \mathrm{~g} \mathrm{~m}^{-2} \sim 240 \mathrm{~kg} \mathrm{~N} \mathrm{ha}^{-1}\right)$ 
En el ciclo I de otoño, se apreció que, a mayor cantidad de abono orgánico aportado, se consiguió un mayor incremento en el peso de las lechugas, pasando de los 250 $\mathrm{g} \mathrm{ud}^{-1}$ con la dosis BL1 a los $300 \mathrm{~g} \mathrm{ud}^{-1}$, con la dosis BL4. El fertilizante mineral y la dosis más baja de $B L$, proporcionaron lechugas de peso parecido (230-240 $\left.\mathrm{g} \mathrm{ud}^{-1}\right)$.

En el ciclo II de invierno, fruto del efecto residual de los abonados realizados durante el ciclo I de otoño, se pudo apreciar como el abono orgánico $\mathrm{BL}$ a las dosis más altas (BL2, BL3 y BL4) dio lugar a lechugas con un peso igual o superior a $100 \mathrm{~g} \mathrm{ud}^{-1}$, peso mínimo que debe presentar una lechuga para poder ser comercializada (Reglamento (CE) $N^{0}$ 1543/2001). Sin embargo, en los bancales control (C) y mineral (M) las lechugas no superaron los $50 \mathrm{~g} \mathrm{ud}^{-1}$. La dosis más alta de $B L(B L 4)$ produjo las lechugas de mayor peso (152 $\left.\mathrm{g} \mathrm{ud}^{-1}\right)$ en este ciclo.

En los dos ciclos de otoño-invierno se observó la misma tendencia: a mayor cantidad de abono orgánico aplicada, mayor peso por unidad de lechuga cosechada (figura 1).

\section{Producción de lechuga: cultivos de primavera}

Al igual que en los ciclos otoño-invierno, los tratamientos fertilizantes dieron lugar a lechugas con pesos medios superiores a las parcelas control, tanto en el ciclo I como en el ciclo II de primavera.

Las lechugas de los tratamientos control (C) fueron las de menor peso, seguidas por las recogidas en los bancales donde se aportó nitrato amónico (figura 2). En el ciclo I de primavera, las mejores producciones de lechuga, $270 \mathrm{~g} \mathrm{ud}-$
1 , se obtuvieron en los bancales que recibieron el fertilizante mineral de liberación lenta (Mr) (figura 2), seguidas de las fertilizadas con las dos dosis más altas de abono orgánico (BL3 y BL5). En el ciclo II de primavera se evidenció el efecto residual de todos los tratamientos de fertilización respecto al control (figura 2), especialmente de los tratamientos con BL. Las lechugas de mayor peso $(470 \mathrm{~g}$ $u^{-1}$ ) se recolectaron en los bancales que habían recibido la dosis más alta de BL (BL5: 1904,0 $\mathrm{g} \mathrm{m}^{-2} \sim 360 \mathrm{~kg} \mathrm{~N}^{-1}$ ) en el ciclo I.

En el ciclo II de primavera se puede observar la misma tendencia que en los ciclos de otoño-invierno, a mayor cantidad de abono orgánico, mayor peso por unidad de lechuga cosechada. Sin embargo, en el ciclo I de primavera prácticamente no hubo diferencias entre las tres dosis de abono orgánico (BL3, BL4 y BL5) (figura 2).

Masarirambi et al. (2012) también encontraron producciones comerciales más altas en el cultivo de lechuga empleando dosis de 20, 40 y 60 t ha-1 de estiércol de pollo fresco, frente al abonado con un fertilizante de síntesis 2:3:2 (Tewolde et al. 2010) + 0,5 de Zn. Ellos explicaron estos resultados debido al importante aporte de $\mathrm{P}$ y K que supone la fertilización con el estiércol de pollo, también debido a la menor retención de humedad del abono inorgánico frente al orgánico y a las conocidas mejoras de las propiedades físicas y químicas del suelo tras el aporte de abonos orgánicos. Estos resultados son acordes con los obtenidos por otros autores que han aplicado estiércol de pollo en cultivos hortícolas (Ghanbarian et al. 2008; Ouda \& Mahadeen, 2008; Xu et al. 2005) y que han probado que se trata de un abono que da lugar a producciones similares o superiores a las obtenidas con fertilización mineral.

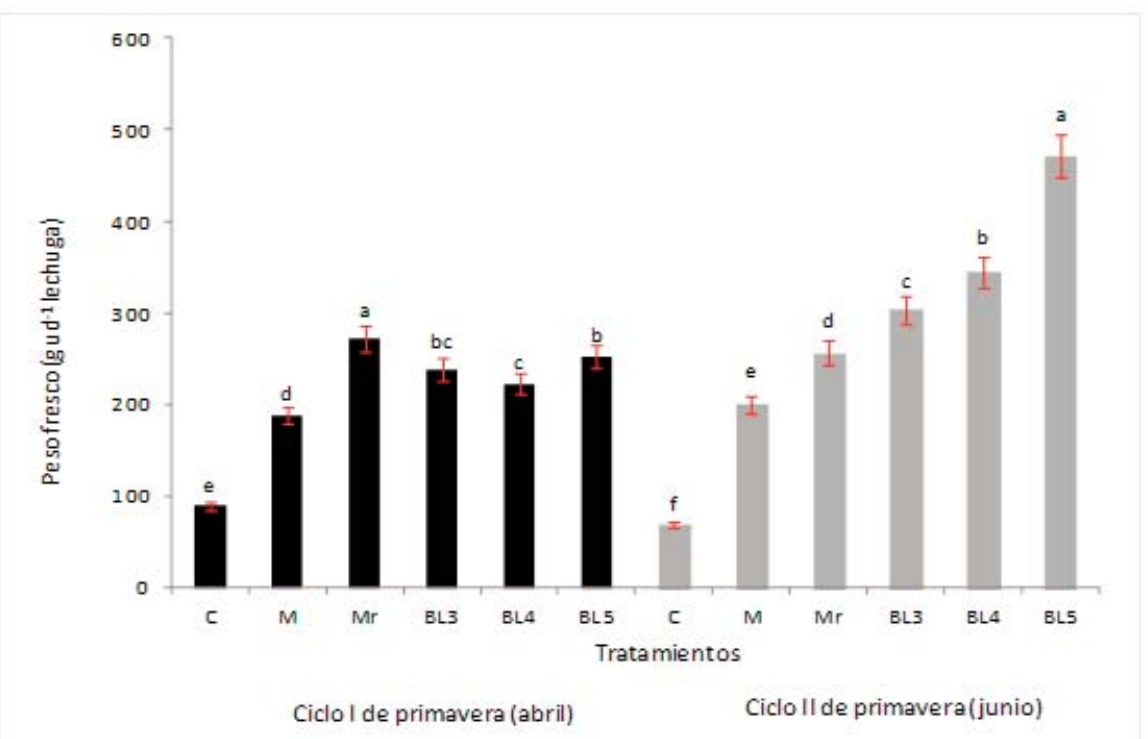

Barras encabezadas por distintas letras indican diferencias significativas entre tratamientos para $p<0,05$.

Figura 2.- Peso fresco de lechuga ( $\mathrm{g} \mathrm{ud}^{-1}$ de lechuga) en los cultivos I y II de primavera. C: control; M: nitrato amónico $\left(58,5 \mathrm{~g} \mathrm{~m}^{-2} \sim 120 \mathrm{~kg} \mathrm{~N}^{-1}\right)$; Mr: fertilizante mineral de liberación lenta $\left(100,0 \mathrm{~g} \mathrm{~m}^{-2} \sim 120 \mathrm{~kg} \mathrm{~N}^{-1}\right)$, estiércol deshidratado y granulado BL, BL3: $\left(634,7 \mathrm{~g} \mathrm{~m}^{-2} \sim\right.$ $\left.120 \mathrm{~kg} \mathrm{~N} \mathrm{ha}^{-1}\right)$, BL4: $\left(1236,9 \mathrm{~g} \mathrm{~m}^{-2} \sim 240 \mathrm{~kg} \mathrm{~N} \mathrm{ha}^{-1}\right)$ y BL5: $\left(1904,0 \mathrm{~g} \mathrm{~m}^{-2} \sim 360 \mathrm{~kg} \mathrm{~N} \mathrm{ha}^{-1}\right)$ 
En el primer ciclo de primavera no se obtuvieron las producciones esperadas en esta época, ni siquiera se observaron diferencias al aumentar las dosis de BL aplicadas. Es posible que la lenta descomposición del estiércol diese lugar a estos resultados. La partida de BL que se utilizó en este ensayo tenía un menor contenido en $\mathrm{N}$ de lo habitual en este producto (3,7\%), lo que obligó a utilizar dosis más elevadas, que tal vez se incorporaron con mayor dificultad al suelo, lo que pudo producir que la flora microbiana fuese aprovechando el abono con lentitud.

Esto mismo observaron los investigadores Hammermeister et al. (2006), que tras haber aplicado altas dosis de estiércol de pollo en cultivo de tomate y lechuga, los rendimientos y el crecimiento de los cultivos fueron bajos, debido en este caso, a la lenta liberación de los nutrientes del estiércol, que necesitó de un periodo de tiempo más largo para transformarse y liberar nutrientes disponibles para las plantas.

La cantidad de $\mathrm{N}$ aportado con el estiércol de pollo depende no solo del contenido total, sino de las formas que dominen en el mismo y de su relación $\mathrm{C} / \mathrm{N}$. La relación $\mathrm{C} / \mathrm{N}$ del $\mathrm{BL}$ era baja $(7,7$ y 11,4$)$ en las dos partidas utilizadas en ambos ensayos, lo que facilitó que una parte importante del $\mathrm{N}$ se liberase durante la primera cosecha. Sin embargo, su alto contenido en N orgánico $(80,8 \%$ ) (Broadley et al. 2003) explica que una parte importante del $\mathrm{N}$ del $\mathrm{BL}$ se pudiese mineralizar y por tanto, estuviese disponible para ser aprovechado por la segunda cosecha, siendo suficiente para satisfacer las necesidades de la lechuga. Estos resultados concuerdan con los presentados por Ribeiro et al. (2010) quienes, pasadas siete semanas tras la aplicación de estiércol de pollo en cultivo de lechuga, comprobaron que la liberación de $\mathrm{N}$ al suelo fue mayor que la ocurrida con otros abonos orgánicos de mayor relación $\mathrm{C} / \mathrm{N}$.
El efecto residual del estiércol de pollo ha sido y es objeto de numerosos estudios. Se ha evaluado su efecto residual sobre la producción de distintos sistemas de cultivo y especies y sobre las propiedades del suelo (Adeli et al. 2011; Tewolde et al. 2011). En ensayos de larga duración Mitchell y Tu (2005) encontraron que fertilizando con estiércol de pollo, se produjeron efectos residuales en el segundo año de aplicación que supusieron incrementos de producción del 30 al $50 \%$ en algodón y del 25 al $65 \%$ en grano de maíz. Adeli et al. (2011) mostraron que tras tres años de aplicación de estiércol de pollo en el cultivo de algodón, se produjeron incrementos de $\mathrm{C}, \mathrm{N}$ y $\mathrm{P}$ en el suelo, además de mejorar la actividad microbiana del mismo, manteniendo su nivel de fertilidad en el tiempo. Destacan además, el potencial que el estiércol de pollo posee frente a los fertilizantes inorgánicos mejorando las propiedades del suelo.

En el ciclo II de primavera se obtuvo una relación cuadrática entre las dosis crecientes de BL y el peso fresco por unidad de lechuga (figura 3 ), siendo el coeficiente de regresión significativo $\left(R^{2}=0,95\right)$. Es decir, el peso fresco de las lechugas aumentó al incrementarse la dosis del estiércol de pollo, aunque no se llegó a alcanzar el punto en el cual a mayor dosis de abono, deje de incrementarse el peso de las lechugas

Para estimar la dosis máxima del abono orgánico $\mathrm{BL}$ a partir de la cual se produce un descenso del peso fresco (g) por unidad de lechuga hubo que recurrir al cálculo sobre la base de modelos cuadráticos ajustados a la ecuación de regresión que se muestra en la figura $3\left(Y=-26,896 x^{2}+\right.$ $259,62 x-150,48)$, donde $x$ representa la cantidad de abono orgánico aportado $\left(\mathrm{g} \mathrm{m}^{-2}\right)$ e $\mathrm{Y}$ equivale al peso fresco $(\mathrm{g})$ por unidad de lechuga.

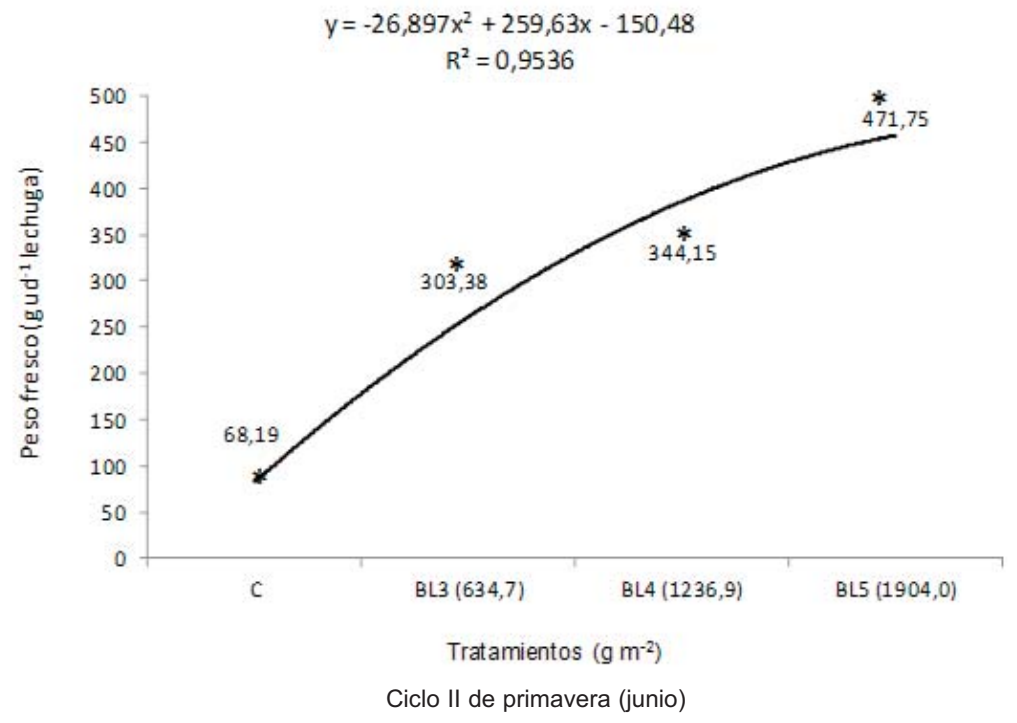

Figura 3.- Peso fresco (g) por unidad de lechuga en función de la dosis de abono orgánico utilizada y ecuación de regresión para las lechugas del segundo ciclo de primavera. C: control; M: nitrato amónico $\left(58,5 \mathrm{~g} \mathrm{~m}^{-2} \sim 120 \mathrm{~kg} \mathrm{~N}^{-1}\right)$; Mr: fertilizante mineral de liberación lenta (100,0 $\left.\mathrm{g} \mathrm{m}^{-2} \sim 120 \mathrm{~kg} \mathrm{~N}^{-1}\right)$; estiércol deshidratado y granulado BL, BL3: (634,7 $\mathrm{g} \mathrm{m}^{-2} \sim 120$ $\left.\mathrm{kg} \mathrm{N} \mathrm{ha}^{-1}\right)$, BL4: (1236,9 $\left.\mathrm{g} \mathrm{m}^{-2} \sim 240 \mathrm{~kg} \mathrm{~N} \mathrm{ha}^{-1}\right)$ y BL5: (1904,0 $\left.\mathrm{g} \mathrm{m}^{-2} \sim 360 \mathrm{~kg} \mathrm{~N}^{-1}\right)$. 
La dosis de BL que daría como resultado una máxima producción sería de $4.826 \mathrm{~g} \mathrm{~m}^{-2}$, para lo cual fue necesario igualar a cero la primera derivada de la ecuación anteriormente expuesta y resolverla para obtener dicha dosis máxima.

En los ensayos realizados en ningún caso se llegó al techo de producción, ni se observaron síntomas en el cultivo que demostrasen excesos de fertilización.

En ensayos de larga duración utilizando estiércol de pollo en cultivo de algodón, Mitchell y Tu (2005) también encontraron que la respuesta a la producción era mejor descrita por modelos cuadráticos, de tal manera que el 95 \% del máximo de producción se originaba con dosis que variaban entre 175 y $193 \mathrm{~kg}$ de $\mathrm{N} \mathrm{ha}^{-1}$. Ajustándose también a modelos cuadráticos, Tewolde et al. (2010) comprobaron que dosis por encima de los $90 \mathrm{~kg} \mathrm{ha}^{-1}$ de $\mathrm{N}$ en forma de nitrato amónico o con dosis de estiércol de pollo superiores a $6,7 \mathrm{Mg} \mathrm{ha}^{-1}$ hacían descender la producción de fibra de algodón y contribuían además a generar problemas ambientales.

\section{Producción comercial en ciclos de otoño-invierno y primavera}

En el ciclo I de otoño los tratamientos que dieron lugar al mayor porcentaje de lechugas comerciales fueron la dosis más alta de estiércol de pollo, BL4 (1064 g m-2 $240 \mathrm{~kg}$ de $\mathrm{N}$ por ha) y el tratamiento con nitrato amónico (90 y $91 \%$ respectivamente), aunque no se encontraron diferencias significativas entre los tratamientos orgánicos y el mineral (figura 4).

En el ciclo I de otoño, una dosis superior a los $80 \mathrm{~kg}$ de $\mathrm{N}$ ha-1 $\left(366,7 \mathrm{~g} \mathrm{~m}^{-2}\right)$ en los bancales abonados con $\mathrm{BL}$, originó una estabilización e incluso un descenso de la producción comercial de un $5 \%$ en las dosis más altas (BL3, 532,0 g m$2 \sim 120 \mathrm{~kg} \mathrm{~N}^{-1}$ y BL4, 1064,0 $\left.\mathrm{g} \mathrm{m}^{-2} \sim 240 \mathrm{~kg} \mathrm{~N} \mathrm{ha}^{-1}\right)$. De modo similar, Rincón et al., en 2002 cosecharon un número de lechugas comerciales inferior cuando la dosis de abonado mineral superó los $150 \mathrm{~kg}$ de $\mathrm{N}$ por hectárea. Esta situación se repitió en el ciclo I de primavera, donde no se encontraron diferencias significativas entre dosis de $\mathrm{BL}$, es decir dosis mayores de $120 \mathrm{~kg}$ de $\mathrm{N}$ por hectárea $(532,0 \mathrm{~g}$ $\left.\mathrm{m}^{-2}\right)$ no mejoraron el porcentaje de lechugas comerciales. Hay que tener en cuenta que dosis elevadas de $\mathrm{N}$ pueden dar lugar a un retraso en la formación del cogollo de la lechuga (Rincón et al. 2002).

En cuanto al ciclo II de invierno, la producción comercial del tratamiento orgánico BL4 (1064 g m-2 $240 \mathrm{~kg}$ de N por ha) fue superior a la de los tratamientos control (C) y mineral (M) en un $71 \%$ y $70 \%$, respectivamente. Dentro de las parcelas abonadas con BL, sólo en los bancales donde se empleó la mayor cantidad de BL (1064 $\mathrm{g} \mathrm{m}^{-2} \sim 240 \mathrm{~kg}$ de $\mathrm{N}$ por ha) se consiguió un porcentaje de lechugas comerciales superior a las no comerciales (figura 4).

Si se compara el ciclo I de otoño con el ciclo II de invierno, el porcentaje de lechugas comerciales se redujo significativamente en el ciclo II en los tratamientos control y mineral, no superando el 4 y el $3 \%$ de lechugas comerciales en estos tratamientos. En las parcelas fertilizadas con BL la reducción fue menor.

En el ciclo I de primavera el fertilizante mineral de liberación lenta (Mr) proporcionó el porcentaje más alto de lechugas comerciales. Los tratamientos orgánicos BL3, BL4 y BL5 produjeron un mayor porcentaje de lechugas comerciales que el tratamiento mineral (M), pero sensiblemente inferior al $\mathrm{Mr}$ (figura 5). Sin embargo, el porcentaje de lechugas comerciales en el ciclo II de primavera fue siempre mayor en los bancales donde se aplicó BL.

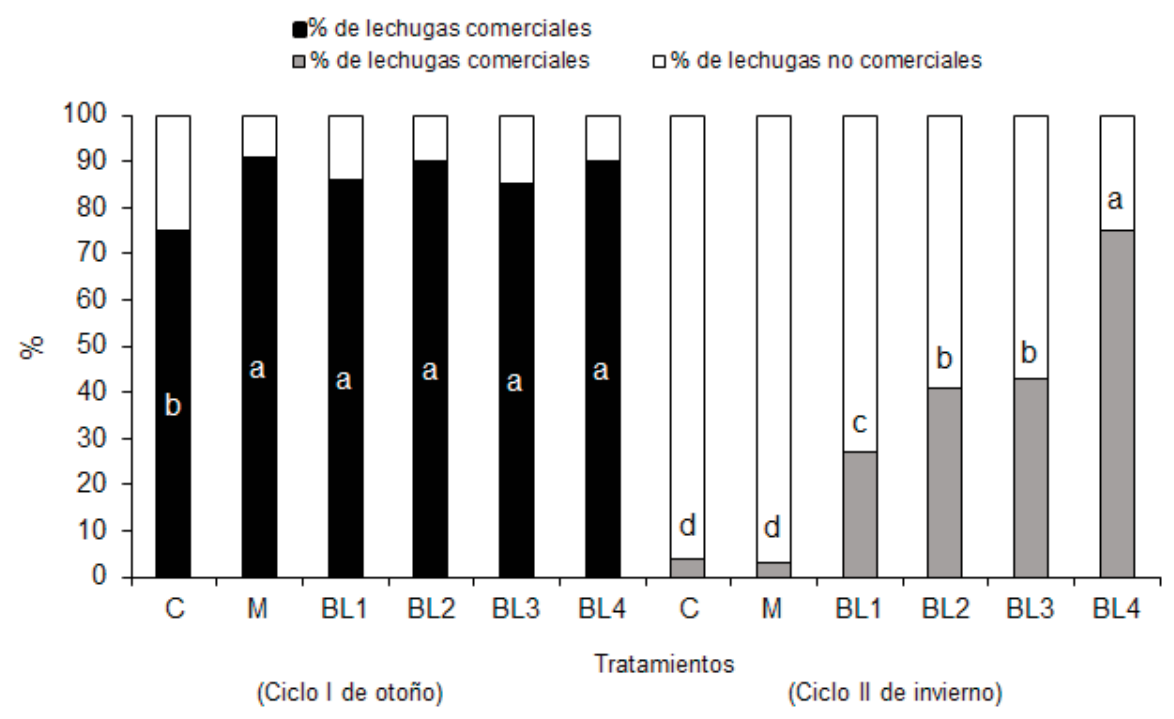

Barras encabezadas por distintas letras indican diferencias significativas entre tratamientos para $p<0,05$.

Figura 4.- Producción comercial y no comercial obtenida según tratamientos en los cultivos de otoño-invierno. C: control; M: Nitrato amónico $\left(58,5 \mathrm{~g} \mathrm{~m}^{-2} \sim 120 \mathrm{~kg} \mathrm{~N} \mathrm{ha}^{-1}\right)$; estiércol deshidratado y granulado BL, BL1: $\left(266,7 \mathrm{~g} \mathrm{~m}^{-2} \sim 60 \mathrm{~kg} \mathrm{~N}^{-1}\right), \mathrm{BL2}:\left(366,7 \mathrm{~g} \mathrm{~m}^{-2} \sim 80 \mathrm{~kg} \mathrm{~N}\right.$ ha-1), BL3: $\left(532,0 \mathrm{~g} \mathrm{~m}^{-2} \sim 120 \mathrm{~kg} \mathrm{~N} \mathrm{ha}^{-1}\right)$ y BL4: $\left(1064,0 \mathrm{~g} \mathrm{~m}^{-2} \sim 240 \mathrm{~kg} \mathrm{~N} \mathrm{ha}^{-1}\right)$ 


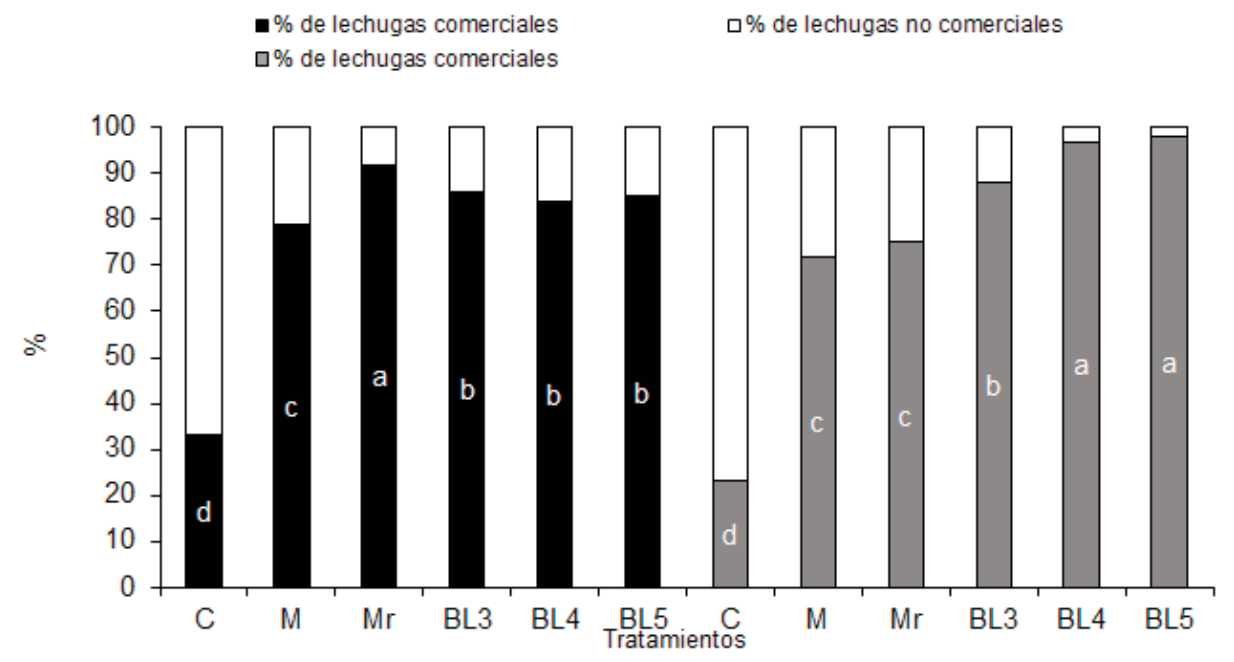

Barras encabezadas por distintas letras indican diferencias significativas entre tratamientos para $p<0,05$.

Figura 5.- Producción comercial y no comercial obtenida según tratamientos en los cultivos de primavera. C: control; M: nitrato amónico . C: control; M: Nitrato amónico $(58,5 \mathrm{~g} \mathrm{~m}-2 \sim 120 \mathrm{~kg} \mathrm{~N}$ ha-1); Mr: fertilizante mineral de liberación lenta (100,0 $\left.\mathrm{g} \mathrm{m}^{-2} \sim 120 \mathrm{~kg} \mathrm{~N} \mathrm{ha}^{-1}\right)$; estiércol deshidratado y granulado BL, BL1: $\left(266,7 \mathrm{~g} \mathrm{~m}^{-2} \sim 60 \mathrm{~kg} \mathrm{~N} \mathrm{ha}^{-1}\right), \mathrm{BL} 2:\left(366,7 \mathrm{~g} \mathrm{~m}^{-2} \sim 80 \mathrm{~kg} \mathrm{~N}^{-}\right.$ 1), BL3: $\left(532,0 \mathrm{~g} \mathrm{~m}^{-2} \sim 120 \mathrm{~kg} \mathrm{~N}^{-1}\right)$ y BL4: $\left(1064,0 \mathrm{~g} \mathrm{~m}^{-2} \sim 240 \mathrm{~kg} \mathrm{~N} \mathrm{ha}^{-1}\right)$

En el ciclo II de invierno, así como en el ciclo II de primavera, el porcentaje de lechugas comerciales en los tratamientos orgánicos fue mucho mayor que en los restantes, invirtiéndose la tendencia anteriormente descrita. El fertilizante BL se comportó como un abono de liberación gradual, liberando un importante porcentaje de nitrógeno en las segundas cosechas fruto del efecto residual del abonado aplicado dos meses antes. Es evidente que la fertilización orgánica con dosis superiores a $120 \mathrm{~kg}$ de $\mathrm{N}$ ha1 en el periodo otoño-invierno $\left(532,0 \mathrm{~g} \mathrm{~m}^{-2}\right)$ y primavera $\left(634,7 \mathrm{~g} \mathrm{~m}^{-2}\right)$ proporcionan nutrientes suficientes para dar un segundo ciclo con un alto porcentaje de lechugas comerciales.

Agradecimientos Los autores agradecen a Juan Carlos Serrano, gerente de la empresa Aviporto S.L., el facilitarnos el facilitarnos el fertilizante BIOF-1. Este trabajo fue financiado por la Dirección General de Investigación del Ministerio de Ciencia y Tecnología (proyecto AGL2000-04-81).

\section{Conclusión}

En los cuatro ciclos de lechuga estudiados, dos en condiciones climáticas más desfavorables (otoño-invierno) y dos en mejores condiciones (primavera), el abonado con $\mathrm{BL}$, a cualquiera de las dosis aplicadas, garantiza la producción del cultivo de lechuga, tanto en peso fresco, como en número de lechugas comerciales, del mismo modo que el abonado mineral.

Se demuestra que el abonado con BL, en este cultivo de huerta en invernadero, resulta competitivo con los fertilizantes inorgánicos ensayados.

En cuanto a las recomendaciones de abonado con BL, el añadirlo para proporcionar 532,0-634,7 $\mathrm{g} \mathrm{m}^{-2}(120 \mathrm{~kg} \mathrm{~N}$ ha-
1) será suficiente para obtener producciones superiores a las que resultan del abonado mineral convencional de lechuga en invernadero y en conjunto similares a las obtenidas con abonos de síntesis de liberación lenta.

El abono BL tiene un efecto residual en la producción de lechuga, tanto en verano como en invierno, que se incrementa con la dosis. Esto repercute en la programación del abonado con este fertilizante, que permite realizar una única aplicación para la obtención de dos cosechas comerciales de este cultivo.

\section{Bibliografía}

Adeli, A.; Sistani, K.R.; Rowe, D.E. \& Tewolde, H. (2005). Effects of broiler litter on soybean production and soil nitrogen and phosphorus concentrations. Agronomy Journal. 97: 314-321.

Adeli, A.; Tewolde, H.; Rowe, D.E. \& Sistani, K.R. (2011). Continous and Residual Effects of Broiler Litter Application to Cotton on Soil Properties. Soil Science. 176, 12: 668675.

Beegle, D. \& Bosworth, J. 1997. Nutrient management. Agron. Facts. 16: 5.

Broadley, R.M.; Seigner, I.; Burns, A.; Escobar-Gutiérrez, A.J.; Burns, I.G.; White, P.J. (2003). The nitrogen and nitrate economy of butterhead lettuce (Lactuca sativa var capitata L.). Journal of Experimental Botany. 390, 54: 2081-2090.

Gascho, G.J. \& Hubbard, R.K. (2006). Long-term impact of broiler litter on chemical properties of a Coastal Plain soil. Journal of Soil and Water Conservation. 61: 65-74. 
Ghanbarian, D.; Youneji, S.; Fallah, S. \& Farhadi, A. (2008). Effect of broiler litter on physical properties, growth and yield of two cultivars of Cantaloupe (Cucumis melo L.). International Journal of Agriculture and Biology. 10 : 697700 .

Hammermeister, A.M.; Astatkie, T.; Jeliazkova, A.; Warman, P.R. \& Martin, R.C. (2006). Nutrient supply from organic amendments applied to unvegetated soil, lettuce and orchardgrass. Canadian Journal of Soil Science. 86, 1: 2123.

Kissel, D.E.; Risse, M.; Sonon, L. \& Harris, G. (2008). Calculating the fertilizer value of broiler litter. University of Georgia, Cooperative Extension Circle C933. Disponible en: http:// pubs.caes.uga.edu/caespubs/pubs/PDF/C933.pdf (20 mayo, 2015).

Lopez-Mosquera M.E.; Cabaleiro F.; Sainz M.J.; LópezFabal A. \& Carral E. (2008). Fertilizing value of broiler litter: Effects of drying and pelletizing. Bioresource Technology. 99, 13: 5626-5633.

MAGRAMA. 2012. Anuario de Estadística Agraria. Ministerio de Agricultura, Alimentación y Medio Ambiente. Disponible e $n$

http://www.magrama.gob.es/es/estadistica/temas/estadpublicaciones/anuario-de-estadistica/2011/default.aspx (20 junio, 2016).

Masarirambi, M.T.; Dlamini, P.; Wahome, P.K. \& Oseni, T.O. (2012). Effects of Chicken Manure on Growth, Yield and Quality of Lettuce (Lactuca sativa L.) "Taina" Undeer a Lath House in a Semi-Arid Sub-Tropical Environment. American-Eurasian. Journal of Agriculture and Environmental Sciences. 12, 3: 399-406.

Mitchell C.C. \& Tu, S. (2005). Long-Term Evaluation of Poultry Litter as a Source of Nitrogen for Cotton and Corn. American Society of Agronomy. Agronomy Journal. 97, 2 : 399-407.

Ouda, B.A. \& Mahadeen, A.Y. (2008). Effects of fertilizers on growth, yield, yield components, quality and certain nutrient contents in Broccoli (Brassica oleracea) International Journal of Agriculture and Biology. 10: 627632.

Pratt, R.G. \& Tewolde, H. (2009). Soil fungal population levels in cotton fields fertilized with poultry litter and their relationships to soil nutrient concentrations and plant growth parameters. Applied Soil Ecology. 41: 41-49.
Reglamento (CE) № 1543/2001. Comisión europea del 27 de junio de 2001.

Ribeiro, H.M.; Fanqueiro, D.; Alves F.; Ventura, R.; Coelho, D.; Vasconcelos, E. ; Cunha-Queda, C.; Coutinho, J. \& Cabral, F. (2010). Nitrogen mineralization from an organically manged soil and nitrogen accumulatin in lettuce. Journal of Plant Nutrition and Soil Science. 173, 2: 260-267.

Rincón, L.; Pérez, A.; Pellicer, C.; Sáez, J. \& Abadía, A. (2002). Influencia de la fertilización nitrogenada en la absorción de nitrógeno y acumulación de nitratos en la lechuga iceberg. Investigaciones Agrarias: Producción y Protección Vegetal. 17: 303-318.

Roberson, T.; Reddy, K.C.; Reddy, S.S.; Nyakatawa, E.Z.; Rape, R.L.; Reeves, D.W. \& Lemunyon, J. (2008). Carbon dioxide efflux from soil with poultry litter application in conventional and conservation tillage systems in northern Alabama. Journal of Environmental Quality. 37: 535-541.

Tewolde, H.; Sistani, K.R. \& Rowe, D.E. (2005a). Broiler litter as a micronutrient source for cotton: Concentrations in plant parts. Journal of Environmental Quality. 34: 16971706.

Tewolde, H.; Sistani, K.R. \& Rowe, D.E. (2005b). Broiler litter as a sole nutrient source for cotton: Nitrogen, phosphorus, potassium, calcium, and magnesium concentrations in plant parts. Journal of Plant Nutrition. 28: 605-619.

Tewolde, H.; Sistani, K.R.; Rowe, D.E.; Adeli, A. \& Johnson, J.R. (2007). Lint yield and fiber quality of cotton fertilized with broiler litter. Agronomy Journal. 99: 184-194.

Tewolde, H.; Adeli, A.; Sistani, K.R.; Rowe, D.E. \& Johnson, J.R. (2010). Equivalency of Broiler Litter to Ammonium Nitrate as a Cotton Fertilizer in an Upland Soil. Agronomy Journal. 102, 1: 251-257.

Tewolde, H.; Adeli, A.; Rowe, D.E. \& Sistani, K.R. (2011). Cotton Lint Yield Improvement Attributed to Residual Effect of Repeated Poultry Litter Application. Agronomy Journal. 103, 1: 107-112.

Xu, H.L.; Wang, R.; Xu, R.Y.; Mridha, M.A.U. \& Goyal, S. (2005). Yield and quality of leafy vegetables grown with organic fertilizations. Acta Horticulturae. 627: 25-33. 